\title{
Accelerated carcinogenesis following liver resection in chronically inflamed livers: A window of opportunity for treatment (Review)
}

\author{
AMIR SONNENBLICK and TAMAR ZAHAVI \\ Sharett Institute of Oncology, Hadassah-Hebrew University Medical Center, Jerusalem 91120, Israel
}

Received December 7, 2016; Accepted February 22, 2017

DOI: 10.3892/br.2017.882

\begin{abstract}
The long-term prognosis following resection of hepatocellular carcinoma $(\mathrm{HCC})$ remains unsatisfactory as a result of a high incidence of recurrence. Prevention of recurrence is the most important strategy to improve the long-term survival results. The role of hepatectomy itself, as an accelerator of carcinogenesis, has not been adequately evaluated in HCC patients. Studies in animal models have revealed a link between liver regeneration under chronic inflammation and hepatic tumorigenesis. Inhibiting different signal transduction pathways during liver regeneration without compromising the ability of the liver to regenerate appears to be a rational strategy and may decrease HCC development and recurrence. If this hypothesis is proven using animal models, this strategy could be evaluated in future clinical trials in humans.
\end{abstract}

\section{Contents}

1. Introduction

2. Different patterns of HCC recurrence

3. Animal models of HCC accelerated carcinogenesis

4. Targeting accelerated carcinogenesis during hepatectomy

5. Future challenges

6. Conclusion

\section{Introduction}

Studies estimate that $>30 \%$ of all malignancies worldwide are initiated or exacerbated by inflammation, and preclinical

Correspondence to: Dr Amir Sonnenblick, Sharett Institute of Oncology, Hadassah-Hebrew University Medical Center, Ein Kerem, P.O. Box 12000, Jerusalem 91120, Israel

E-mail: amirsonn@gmail.com

Abbreviations: HCC, hepatocellular carcinoma; KO, knockout; $\mathrm{PH}$, partial hepatectomy; STAT3, signal transducer and activator of transcription 3

Key words: hepatocellular carcinoma, hepatectomy, sorafenib, signal transducer and activator of transcription 3, inflammation data also supports the concept that inflammation is a pivotal component of tumor initiation (1). Hepatocellular carcinoma (HCC), the third leading cause of cancer mortality worldwide, commonly develops in an inflamed liver following a prolonged chronic hepatitis state (2). Hepatic resection and liver transplantation are the only treatments with curative intent for $\mathrm{HCC}$, where resection as a bridge to transplantation is also emerging as a possible therapeutic strategy (3-6). For patients with early HCC and decompensated cirrhosis, liver transplantation is the treatment of choice, as the procedure potentially cures the cirrhosis and the HCC, and the outcome is accepted to be better than that of hepatic resection (7). HCC is most prevalent in areas with endemic viral hepatitis $\mathrm{B}$ or $\mathrm{C}$, which is typical in many African and Asian countries (8). In these endemic countries transplantation procedures are limited due to a shortage of living donors, legal and economic issues and lack of resources. Therefore, hepatic resection is considered to be the practical curative treatment option for many patients with HCC globally.

\section{Different patterns of HCC recurrence}

Despite improved resection techniques, and subsequent decreased operative morbidity and mortality in hepatic surgery, survival rates following partial hepatectomy $(\mathrm{PH})$ are suboptimal, predominantly due to tumor recurrence, which within five years occurs in the range of 75 to $100 \%$ of cases $(9,10)$. It was estimated that $60-70 \%$ of recurrences were attributed to intrahepatic lesions undetected at the time of resection, whereas $30-40 \%$ were de novo HCCs (Fig. 1) (11,12). The two different patterns of recurrence are proposed to represent distinct carcinogenesis patterns that have markedly different prognoses. Early recurrence usually represents residual tumor spread from the primary main tumor and remains in the remnant liver, which is an indicator of a poor prognosis. Significant risk factors for early recurrence include preoperative tumor rupture, venous invasion and non-anatomic resection $(13,14)$. Late recurrence usually results from multicentric hepatocarcinogenesis. Possible risk factors for late recurrence include cirrhosis, higher grade of hepatitis activity and multiple tumors $(11,14,15)$. In certain studies from areas with endemic viral hepatitis, the majority of the recurrences were multicentric in location and distant from the resection margin (16-18). In one study it was found that there was no significant difference in the recurrence rate 
that occurred between major resection and minor or localized resection of the liver (16). This indicated that in these patients the prominent pattern of failure was de novo HCCs. This hypothesis was further confirmed in a study where it was demonstrated that gene expression profiling from liver tissue adjacent to the tumor was correlated with survival in contrast to the genome signature of the tumor itself (19). Therefore, patients with de novo $\mathrm{HCC}$ recurrence, post-curative $\mathrm{PH}$, are the patients that may benefit from inhibition of the accelerated carcinogenesis following liver resection.

\section{Animal models of HCC accelerated carcinogenesis}

HCC in rodents and humans share common features, and various mouse models of this disease have been investigated to establish the underlying molecular mechanisms of liver cancer (20). Few animal studies investigating the effects of liver regeneration on tumor progression were performed using transplanted tumor cells (subcutaneously or directly into the liver), or using chemically induced tumors (21-23). In these animal models, $\mathrm{PH}$ was shown to affect and enhance the initiation and promotion phases of carcinogenesis when compared with sham surgery. However, these models have no underlying liver inflammation, as is the case in the majority of humans with HCC. Therefore, until recently, there was insufficient information regarding the mechanisms by which the inflammatory microenvironment affects liver regeneration, and the effect of inflammation and regeneration on hepatocarcinogenesis.

The Mdr2-knockout (KO) mouse is a model with similarities to human HCC. These mice lack the Mdr2 P-glycoprotein, which is responsible for phosphatidylcholine transport across the canalicular membrane. The absence of phospholipids from bile leads to portal inflammation and slowly developing HCC, which closely mimics the human disease in this regard $(24,25)$. In the Mdr2-KO mice model Barash et al (26) demonstrated that $\mathrm{PH}$, prior to the development of HCC, led to enhanced hepatocarcinogenesis. It was proposed that under the regenerative proliferative stress induced by liver resection in these mice, the genomic unstable hepatocytes, generated during chronic inflammation, escape senescence and apoptosis, and reenter the cell cycle, triggering enhanced tumorigenesis (26).

\section{Targeting accelerated carcinogenesis during hepatectomy}

If $\mathrm{PH}$ potentially accelerates carcinogenesis, it may be worthy to attempt to block potential signaling pathways specifically during this procedure. Numerous cytokines, growth factors and signal transduction pathways are activated during liver regeneration, and some of these may present as potential targets for prevention of accelerated carcinogenesis, although regeneration may be compromised (Fig. 1) (27).

Sorafenib, a mulitkinase inhibitor, is the only systemic therapeutic modality that significantly prolonged the survival of HCC patients with advanced-stage disease and is considered the standard of care for patients with metastatic or locally advanced HCC $(28,29)$. In a study that used an orthotropic HCC model, sorafenib treatment over a short duration subsequent to $\mathrm{PH}$ suppressed accelerated tumor growth (30). It was further demonstrated that postoperative activation of the

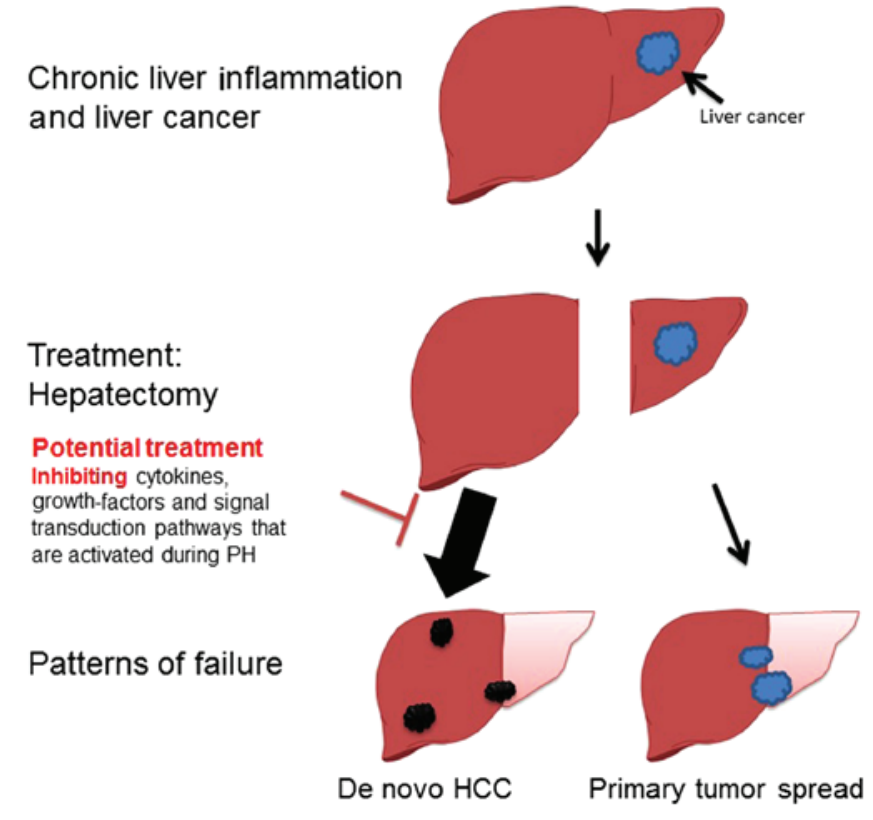

Figure 1. Blocking different signal transduction pathways specifically during $\mathrm{PH}$ could present potential targets for prevention of accelerated carcinogenesis. PH, partial hepatectomy.

Raf-MEK-extracellular signal-regulated kinase (ERK) signal transduction pathway sensitizes HCC to sorafenib. Another preclinical study demonstrated that sorafenib treatment around $\mathrm{PH}$ did not impact liver regeneration when administered prior to surgery; however, administration following $\mathrm{PH}$ reduced liver regeneration (31). Taken together, these studies indicate that short duration sorafenib treatment surrounding $\mathrm{PH}$ for early-stage $\mathrm{HCC}$ is a promising approach for preventing recurrence. However, these studies do not capture the same scenario as in humans, where it is speculated that, under the regenerative proliferative stress induced by $\mathrm{PH}$, the non-tumoral hepatocytes escape senescence and apoptosis, triggering enhanced tumorigenesis.

While sorafenib inhibits the Raf-ERK/platelet-derived growth factor receptor (GFR)/vascular endothelial GFR signaling pathways, other pathways that are activated during liver regeneration and are crucial for HCC development may be targetable. Signal transducer and activator of transcription 3 (STAT3) and its activating cytokine, interleukin (IL)-6 are key regulators of liver regeneration and act to prime hepatocytes to transition from the G0 phase and progress to the G1/S phase $(32,33)$. Thus, the IL-6/STAT3 signaling pathway may be of central importance to the development and progression of HCC following PH. As many therapeutic agents that target the IL-6/STAT3 pathway were recently developed (34) such an approach appears reasonable, although it may be the case that inhibition of the IL-6/STAT3 axis is detrimental to liver regeneration. In a recent study by Zahavi et al (35) it was shown, using the Mdr2-KO mice model, that sorafenib treatment during $\mathrm{PH}$ inhibited various signal transduction pathways at the multikinase levels, which did not result in increased morbidity or mortality. In the early stages subsequent to $\mathrm{PH}$, sorafenib treatment resulted in decreased stellate cell activation and inflammatory response. Finally, it was demonstrated that sorafenib treatment during $\mathrm{PH}$ at 3 months 
of age resulted in decreased fibrosis, and tumor formation at age 8.5 months (35). The study confirmed the hypothesis that short-term treatment during $\mathrm{PH}$ is feasible and effective in inhibiting inflammation-associated cancer, and is therefore a potential strategy for recurrence prevention (35).

\section{Future challenges}

There are two major challenges that will be encountered when evaluating the strategy of treatment using signal transduction blockade during hepatic resection in patients with HCC under inflamed conditions. The first issue includes the high perioperative morbidity and the low survival rate with mortality up to $20 \%$ surrounding surgery (36). Using molecular inhibitors, which may inhibit recovery and decelerate the regeneration of the liver, could be devastating for certain patients and result in mortality; therefore, are not accepted as legitimate in clinical trial design. The second issue is the fact that major, sponsored, randomized clinical trials will not be performed in this context, as the treatment duration is very short (surrounding the surgery) and the potential benefit to the pharmaceutical industry is limited. In order to overcome the lack of incentive of the pharmaceutical companies, such studies require sponsorship from academic institutions.

\section{Conclusion}

Oncologists have long recognized that in certain cancers, surgical treatment demonstrates carcinogenic potential. In the current review, the latest evidence of the potential carcinogenesis of hepatectomy for the treatment of HCC is described. In addition, potential treatment strategies for decreasing this accelerated carcinogenesis were described. Different signal transduction pathway inhibitors are already available and are currently used in many clinical trials for different indications, such as metastatic cancers. Despite the risk to fragile patients with HCC and liver inflammation, and despite the low potential for financial gain, further clinical research using different relevant signaling pathway inhibitors during hepatectomy is encouraged.

\section{Acknowledgements}

The present study was supported by the Morasha program of the Israel Science foundation (grant no. 1728/11) and the Israel cancer association (grant no. 2014-0001).

\section{References}

1. Coussens LM and Werb Z: Inflammation and cancer. Nature 420 : 860-867, 2002

2. Berasain C, Castillo J, Perugorria MJ, Latasa MU, Prieto J and Avila MA: Inflammation and liver cancer: New molecular links. Ann N Y Acad Sci 1155: 206-221, 2009.

3. Llovet JM, Fuster J and Bruix J; Barcelona Clinic Liver Cancer (BCLC) Group: Intention-to-treat analysis of surgical treatment for early hepatocellular carcinoma: Resection versus transplantation. Hepatology 30: 1434-1440, 1999.

4. Adam R, Azoulay D, Castaing D, Eshkenazy R, Pascal G, Hashizume K, Samuel D and Bismuth H: Liver resection as a bridge to transplantation for hepatocellular carcinoma on cirrhosis: A reasonable strategy? Ann Surg 238: 508-518, discussion 518-519, 2003.
5. Marrero JA: Multidisciplinary management of hepatocellular carcinoma: Where are we today? Semin Liver Dis 33 (Suppl 1): S3-S10, 2013.

6. Livraghi T, Mäkisalo H and Line PD: Treatment options in hepatocellular carcinoma today. Scand J Surg 100: 22-29, 2011.

7. Mazzaferro V, Bhoori S, Sposito C, Bongini M, Langer M, Miceli R and Mariani L: Milan criteria in liver transplantation for hepatocellular carcinoma: An evidence-based analysis of 15 years of experience. Liver Transpl 17 (Suppl 2): S44-S57, 2011.

8. El-Serag HB: Epidemiology of viral hepatitis and hepatocellular carcinoma. Gastroenterology 142: 1264-1273.e1, 2012.

9. Schwartz M, Roayaie S and Konstadoulakis M: Strategies for the management of hepatocellular carcinoma. Nat Clin Pract Oncol 4: 424-432, 2007.

10. Llovet JM: Updated treatment approach to hepatocellular carcinoma. J Gastroenterol 40: 225-235, 2005.

11. Poon RT-P, Fan ST, Lo CM, Liu CL and Wong J: Long-term survival and pattern of recurrence after resection of small hepatocellular carcinoma in patients with preserved liver function: Implications for a strategy of salvage transplantation. Ann Surg 235: 373-382, 2002.

12. Yamamoto J, Kosuge T, Takayama T, Shimada K, Yamasaki S, Ozaki H, Yamaguchi N and Makuuchi M: Recurrence of hepatocellular carcinoma after surgery. Br J Surg 83: 1219-1222, 1996.

13. Imamura H, Matsuyama Y, Tanaka E, Ohkubo T, Hasegawa K, Miyagawa S, Sugawara Y, Minagawa M, Takayama T, Kawasaki S, et al: Risk factors contributing to early and late phase intrahepatic recurrence of hepatocellular carcinoma after hepatectomy. J Hepatol 38: 200-207, 2003.

14. Poon RT,Fan ST,Ng IO,Lo CM,Liu CL and Wong J: Different risk factors and prognosis for early and late intrahepatic recurrence after resection of hepatocellular carcinoma. Cancer 89: 500-507, 2000.

15. Ercolani G, Grazi GL, Ravaioli M, Del Gaudio M, Gardini A, Cescon M, Varotti G, Cetta F and Cavallari A: Liver resection for hepatocellular carcinoma on cirrhosis: Univariate and multivariate analysis of risk factors for intrahepatic recurrence. Ann Surg 237: 536-543, 2003.

16. Abdel Wahab M, Sultan A, el-Ghawalby N, Fathy O, Abu Zeid M, Abu el-Enin A, Abdallah T, Foad A, Kandeel T, el-Shobari M, et al: Hepatic resection in cirrhotic liver for treatment of hepatocellular carcinoma in Egyptian patients. Experience with 140 cases in a single center. Hepatogastroenterology 51: 559-563, 2004.

17. Kumada T, Nakano S, Takeda I, Sugiyama K, Osada T, Kiriyama S, Sone Y, Toyoda H, Shimada S, Takahashi M, et al: Patterns of recurrence after initial treatment in patients with small hepatocellular carcinoma. Hepatology 25: 87-92, 1997.

18. Adachi E, Maeda T, Matsumata T, Shirabe K, Kinukawa N, Sugimachi $\mathrm{K}$ and Tsuneyoshi $\mathrm{M}$ : Risk factors for intrahepatic recurrence in human small hepatocellular carcinoma. Gastroenterology 108: 768-775, 1995.

19. Hoshida Y, Villanueva A, Kobayashi M, Peix J, Chiang DY, Camargo A, Gupta S, Moore J, Wrobel MJ, Lerner J, et al: Gene expression in fixed tissues and outcome in hepatocellular carcinoma. N Engl J Med 359: 1995-2004, 2008.

20. Farazi PA and DePinho RA: Hepatocellular carcinoma pathogenesis: From genes to environment. Nat Rev Cancer 6: 674-687, 2006 .

21. Picardo A, Karpoff HM, Ng B, Lee J, Brennan MF and Fong Y: Partial hepatectomy accelerates local tumor growth: Potential roles of local cytokine activation. Surgery 124: 57-64, 1998.

22. de Jong KP, Lont HE, Bijma AM, Brouwers MA, de Vries EG, van Veen ML, Marquet RL, Slooff MJ and Terpstra OT: The effect of partial hepatectomy on tumor growth in rats: In vivo and in vitro studies. Hepatology 22: 1263-1272, 1995.

23. Asaga T, Suzuki K, Umeda M, Sugimasa Y, Takemiya S and Okamoto T: The enhancement of tumor growth after partial hepatectomy and the effect of sera obtained from hepatectomized rats on tumor cell growth. Jpn J Surg 21: 669-675, 1991.

24. Lammert F, Wang DQ-H, Hillebrandt S, Geier A, Fickert P, Trauner M, Matern S, Paigen B and Carey MC: Spontaneous cholecysto- and hepatolithiasis in Mdr2-/- mice: A model for low phospholipid-associated cholelithiasis. Hepatology 39: 117-128, 2004.

25. Mauad TH, van Nieuwkerk CM, Dingemans KP, Smit JJ, Schinkel AH, Notenboom RG, van den Bergh Weerman MA, Verkruisen RP, Groen AK, Oude Elferink RP, et al: Mice with homozygous disruption of the mdr2 P-glycoprotein gene. A novel animal model for studies of nonsuppurative inflammatory cholangitis and hepatocarcinogenesis. Am J Pathol 145: 1237-1245, 1994. 
26. Barash H, R Gross E, Edrei Y, Ella E, Israel A, Cohen I, Corchia N, Ben-Moshe T, Pappo O, Pikarsky E, et al: Accelerated carcinogenesis following liver regeneration is associated with chronic inflammation-induced double-strand DNA breaks. Proc Natl Acad Sci USA 107: 2207-2212, 2010.

27. Taub R: Liver regeneration: From myth to mechanism. Nat Rev Mol Cell Biol 5: 836-847, 2004.

28. Llovet JM, Ricci S, Mazzaferro V, Hilgard P, Gane E, Blanc JF, de Oliveira AC, Santoro A, Raoul JL, Forner A, et al; SHARP Investigators Study Group: Sorafenib in advanced hepatocellular carcinoma. N Engl J Med 359: 378-390, 2008.

29. Cheng A-L, Kang Y-K, Chen Z, Tsao CJ, Qin S, Kim JS, Luo R, Feng J, Ye S, Yang TS, et al: Efficacy and safety of sorafenib in patients in the Asia-Pacific region with advanced hepatocellular carcinoma: A phase III randomised, double-blind, placebo-controlled trial. Lancet Oncol 10: 25-34, 2009.

30. Feng Y-X, Wang T, Deng Y-Z, Yang P, Li JJ, Guan DX, Yao F, Zhu YQ, Qin Y, Wang H, et al: Sorafenib suppresses postsurgical recurrence and metastasis of hepatocellular carcinoma in an orthotopic mouse model. Hepatology 53: 483-492, 2011.
31. Hora C, Romanque P and Dufour JFF: Effect of sorafenib on murine liver regeneration. Hepatology 53: 577-586, 2011.

32. Cressman DE, Greenbaum LE, DeAngelis RA, Ciliberto G, Furth EE, Poli V and Taub R: Liver failure and defective hepatocyte regeneration in interleukin-6-deficient mice. Science 274 : 1379-1383, 1996.

33. Cressman DE, Diamond RH and Taub R: Rapid activation of the Stat 3 transcription complex in liver regeneration. Hepatology 21: 1443-1449, 1995.

34. Sansone P and Bromberg J: Targeting the interleukin-6/Jak/stat pathway in human malignancies. J Clin Oncol 30: 1005-1014, 2012.

35. Zahavi T, Lanton T, Divon MS, Salmon A, Peretz T, Galun E, Axelrod JH and Sonnenblick A: Sorafenib treatment during partial hepatectomy reduces tumorgenesis in an inflammation-associated liver cancer model. Oncotarget 7: 4860-4870, 2016.

36. Wei AC, Tung-Ping Poon R, Fan ST and Wong J: Risk factors for perioperative morbidity and mortality after extended hepatectomy for hepatocellular carcinoma. Br J Surg 90: 33-41, 2003. 